Research Article

\title{
Bending and Buckling Analyses of Composite Laminates with and without Presence of Damage and its Passive Control with Optimized Piezoelectric Patch Location
}

\author{
DIPAK KUMAR MAITI ${ }^{1}$ and V M SREEHARI ${ }^{2}$ \\ ${ }^{1}$ Professor, Department of Aerospace Engineering, Indian Institute of Technology Kharagpur, WB 721302, \\ India \\ ${ }^{2}$ Research student, Department of Aerospace Engineering, IIT, Kharagpur, WB 721302, India
}

(Received on 15 April 2016; Accepted on 25 April 2016)

\begin{abstract}
This work presents an efficient technique to enhance the bending and buckling characteristics of a smart composite plate. This paper discusses about the employment of piezoelectric fibre composite patches (PFCP) in their optimized location using unified particle swarm optimization (UPSO) for enhancing the performance and thereby reducing the effects of internal flaws. A finite element formulation based on Inverse Hyperbolic Shear Deformation Theory (IHSDT) for handling bending and buckling analysis of a smart composite plate is used in the present work. In addition to the best performance, reduction in weight of piezoelectric material is obtained as we employ a segmented piezo patch to overcome the degradation in buckling strength due to damage in a composite plate, which indeed addresses the design issues.
\end{abstract}

Keywords: Composite Plate; Finite Element Method; Piezoelectric Fibre Composites; Optimization

\section{Introduction}

Researchers had a keen interest in the area of smart structures in the past (Crawley, 1987; Hwang and Park, 1993; Chandrashekhara and Bhatia, 1993; Ray et al., 1994; Samanta et al., 1996; Reddy, 1999; Zhou, 2001; Maiti and Sinha, 2011; Wankhade and Bajoria, 2012). The main reasons behind it are limitations in weight, space, and positioning in many applications. Active Fiber Composites (AFCs) was made from the researches at MIT in 1992. AFCs contain PZT (lead zirconate titanate) fibers and epoxy resin. For the purpose of poling and to direct the electric field along the longitudinally oriented PZT fibers, interdigitated electrodes (IDEs) are used. Understanding the superiorities of PFC material to existing actuators PFCs became a significant focus of a number of researchers. Broad elementary research into various aspects of AFCs like modelling, manufacturing, and physical incorporation into structures are currently going on. Some advantages of AFCs over monolithic ceramic actuators are conformability to curved surfaces, high performance, manufacturability, increased robustness to damage, etc. Specific strength and directional sensitivity of fine ceramic fibers are higher than monolithic materials. A detailed study on AFC properties can be seen in literature (Bent, 1997). But, some weaknesses may arise in their application, say, when composite structure experiences large deformation and/or the surface of the composite structure is geometrically nonconformable. In such cases fibers may break (because they are thin, brittle and continuous piezoelectric). Subsequently it will affect the actuation capability of actuator. So an effective method is to use these piezoelectric fibre actuators in the form of patch instead of complete layer.

For solving any physical system, firstly it is transformed into a mathematical model using some technique. Then any solution technique is used to solve the mathematical model. The accuracy of any 


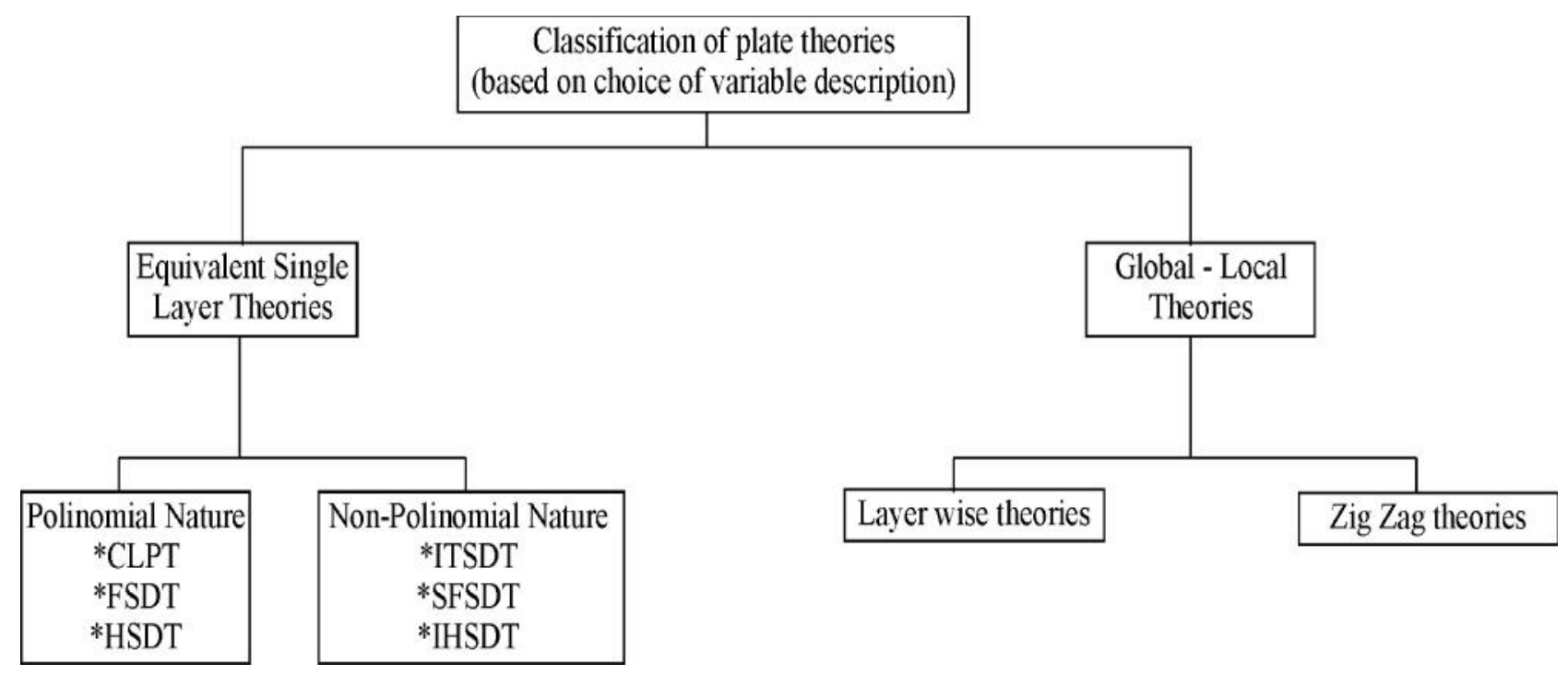

Fig. 1: Classification of plate theories based on choice of variable description

mathematical model depends mainly upon above transformation and solution techniques. For getting the exact responses three-dimensional (3D) elasticity methods can be employed. But 3D solutions can be used only for specific boundary and geometry conditions. Due to above reason, it is better to use two-dimensional (2D) models in the investigation of composite structures. Because of the large ratio of elastic modulus to shear modulus, effects of shear deformation are noteworthy in composite structures and therefore it plays an important role in modelling composite structures. Many plate theories are present which combine the effects of shear deformation in distinctive ways. These are categorized as classical laminated plate theory (CLPT), first order shear deformation theory (FSDT) and higher order shear deformation theory (HSDT) where all these theories have polynomial nature. Over the past few years, various shear deformation theories having nonpolynomial nature and expressed in terms of shearstrain function have been proposed. Some of the nonpolynomial higher-order theories were proposed by Mantari et al. (2012a, 2012b), Karama et al. (2009), Meiche et al. (2011), and Aydogdu (2009). Recently, Grover et al. (2013) proposed new nonpolynomial shear deformation theories and implemented for structural responses of laminated composite and sandwich plates. They explained sheardeformation theory based upon secant function (SDTSF), an inverse-trigonometric shear-deformation theory (ITSDT), and an inverse hyperbolic shear deformation theory (IHSDT) and concluded that it shows improved performance similar to all prevailing higher order shear deformation theories involving shear strain function. A brief classification of plate theories based on choice of variable description is shown in Fig. 1.

Damage can be said as a function of variations in the material properties, geometrical properties, boundary conditions, and structure connectivity, which harmfully affect the present or future performance of structure (Sohn et al., 2004). We have to consider existence, prediction of location, and prediction of the amount or extent for the effective damage evaluation of structures. Therefore, at foremost, the crucial concern of structural damage assessment is to analyse the state of the structure by comparing the response measurements of damaged and undamaged structures. Researchers (Prabhakara and Datta, 1993; Rahul and Datta, 2013) have examined the vibration and parametric instability characteristics in damaged plate in beam structures, where the formulation considers the in-plane membrane effect of the plate in the beam problem. They used a parametric model of damage which was proposed by Valliappan et al. (1990). A continuous parameter that can be correlated to the density of defects is presented in general continuum damage mechanics. This technique is useful in describing the weakening of the material 


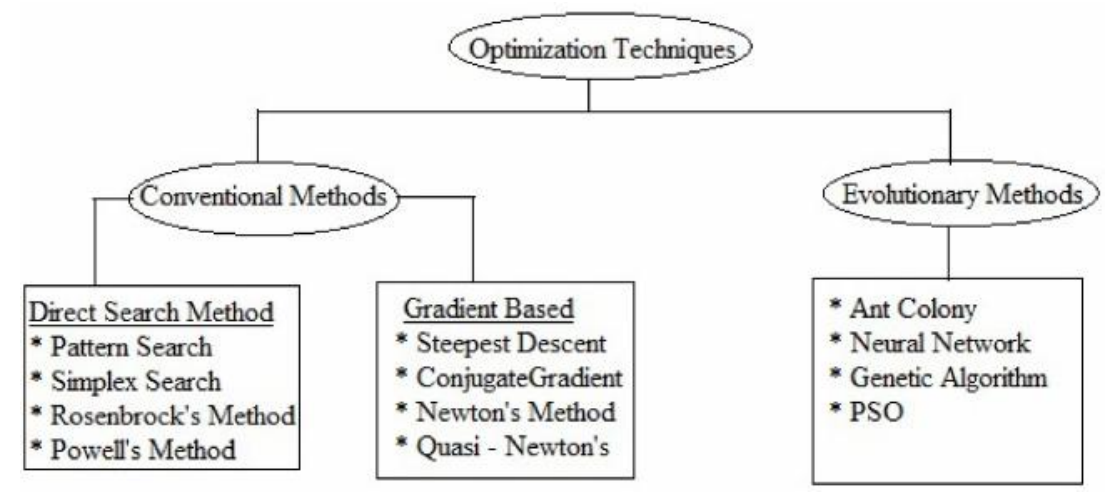

Fig. 2: Classification of optimization methods

before the initiation of micro-cracks. Studies by Pidaparti (1997), Zhang et al. (2001), etc. established that the formulation by Valliappan et al. (1990) has more influence than some other and appreciated that a parametric model is convenient in framing problems related to structural health monitoring.

Regarding the thermal buckling, it is important to explore few significant works done in this area. Tauchert (1987), Tauchert and Huang (1987), Thangaratnam et al. (1989), Chang and Huang (1991), Meyers and Hyer (1991), Noor et al. (1993), Singha et al. (2001), etc. have conducted structural analysis under thermal environment using finite element method.

Many literatures are available describing various optimization methods. A few methods are listed in Fig. 2 and the selection of an appropriate one depends on the nature of problem considered. The particle swarm optimization (PSO) algorithm was first proposed by Kennedy and Eberhart (1995). Parsopoulos and Vrahatis (2002) presented a review of recent results concerning the Particle Swarm Optimization (PSO) method. They concluded that PSO seems to be a very beneficial method and a worthy substitute in cases where other methods fail. Reviews on PSO were conducted by Banks et al. (2007 and 2008). Background and progress of PSO were discussed and recommended the requirement for hybridization to avoid some demerits such as swarm stagnation and dynamic environments. Hybridization of PSO and a variety of application areas are discussed in second article. It is concluded that PSO is greatly effective and flexible to different domain problems and its potential for incorporation with intelligent systems. Many advantages compared to other algorithms make PSO a perfect method to be employed in optimization problems. PSO provides faster results compared with many other optimization methods like the genetic algorithm (Pratihar, 2008; Rao, 2009; Mohan et al., 2014). Currently researchers are using optimization methods for enhancing the performance of smart structures. Frecker (2003) reviewed the works done related to optimization analysis in smart structures design since 1999. Correia et al. (2003) obtained the optimal location of piezoelectric actuators (PZT) and also the optimal fiber reinforcement angles using simulated annealing optimization algorithm. Finite element models using higher order shear deformation theories were used. Yet an optimization analysis of smart structures employing PSO has not got much attention in past.

Structures should be able to survive maximum probable forces acting on them and overcome the effects of minor damages arising in them. We can use smart materials along with structural components to make them survive more forces than what they are expected to. In the present investigation we apply piezoelectricity to strengthen structures, thereby controlling the deformations and increasing the critical buckling load. Also the optimal locations for PFCPs are found out for a composite plate with and without damage.

\section{Mathematical Formulation}

\section{Introduction}

Consider a laminated composite plate having dimensions and geometry as in Fig. 3, where $(x, y, z)$ 


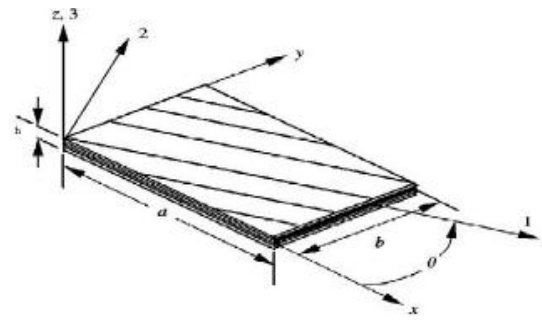

Fig. 3: Dimensions and cross-sectional geometry of the plate

represents the rectangular Cartesian coordinate system. The plane $z=0$ coincides with the midsurface of the plate. A finite element formulation is developed for handling bending and buckling analysis of laminated composite plates with damage. The fundamental idea of finite elements is that the structure is considered as an assembly of elements connected at nodes. An isoparametric element has an advantage that element geometry and displacements are represented by same set of shape functions. In the present analysis 8-noded isoparametric plate element is employed for the analysis. The benefit of 8 noded element is that all the nodes are located on element sides and hence there are no internal nodes and shape functions have quadratic variation in $\mathrm{x}$ and $\mathrm{y}$ direction.

\section{Displacement and Potential Fields}

Authors had explained the buckling of composite structures earlier (Sreehari and Maiti, 2015a). The chosen displacement field for structural analysis of the piezo attached laminated composite plate is on the basis of IHSDT given by:

$$
\begin{aligned}
u(x, y, z)= & u_{0}(x, y)-z \frac{\partial w_{0}}{\partial x} \\
+ & {\left[\sinh ^{-1}(r z / h)-z\left(\frac{2 r}{h \sqrt{\left(r^{2}+4\right)}}\right)\right] \theta_{x} } \\
v(x, y, z)= & v_{0}(x, y)-z \frac{\partial w_{0}}{\partial y} \\
& +\left[\sinh ^{-1}(r z / h)-z\left(\frac{2 r}{h \sqrt{\left(r^{2}+4\right)}}\right)\right] \theta_{y}
\end{aligned}
$$

$w(x, y, z)=w_{0}(x, y)$
In the above displacement field, $u_{0}, v_{0}$ and $w_{0}$ are the midplane displacements while $\theta_{x}, \theta_{y}$ are the shear displacements. The above $\mathrm{C}^{1}$ continuity displacement is converted to $\mathrm{C}^{0}$ continuity after assigning independent field variables to the first derivative of transverse displacement (Sreehari and Maiti, 2015a). The electric potential field of the piezoelectric patches are assumed to be given by:

$$
\phi(x, y, z, t)=\frac{z-h_{k}}{h_{k+1}-h_{k}} \phi_{0}(x, y, t)
$$

The $z$ co-ordinates of laminates corresponding to the top and bottom surface of layer $k$ relative to the midplane are denoted by $h_{k}$ and $h_{k+1}$. In a $k^{\text {th }}$ layer, the coupling effect due to the attachment of piezoelectric patches results in both mechanical and electrical excitation. This can be expressed in terms of stress and electrical displacement using the direct and converse piezoelectric effect as:

$$
\begin{aligned}
& \{D\}_{k}=[e]_{k}^{T}\{\varepsilon\}_{k}+[K]_{k}\{E\}_{k} \\
& \{\sigma\}_{k}=[\bar{Q}]_{k}\{\varepsilon\}_{k}+[e]_{k}\{E\}_{k}
\end{aligned}
$$

The subscript $k$ denotes the layer number. Here $\{D\}$ is the electric displacement vector, $[\bar{Q}]$ is the transformed reduced elastic stiffness matrix, $[K]$ is permittivity coefficient matrix, $\{\sigma\}$ is stress vector and $[e]$ is piezoelectric constant matrix.

$$
\begin{aligned}
\text { where }[e]_{P Z T}^{T}= & {\left[\begin{array}{ccccc}
0 & 0 & 0 & 0 & e_{15} \\
0 & 0 & 0 & e_{24} & 0 \\
e_{31} & e_{32} & 0 & 0 & 0
\end{array}\right] } \\
\text { and }[e]_{P F C}^{T} & =\left[\begin{array}{ccccc}
e_{11} & e_{12} & 0 & 0 & 0 \\
0 & 0 & 0 & e_{24} & 0 \\
0 & 0 & 0 & 0 & e_{35}
\end{array}\right]
\end{aligned}
$$

\section{Governing Equation}

The global governing dynamic equation of the piezoelectric attached laminated composite plate as for bending control analysis can be modified and can be represented globally as: 


$$
\begin{aligned}
{\left[K_{u u}+\gamma K_{c}\right]\{u\} } & =\left\{F_{1}\right\}+\left[K_{u \phi}\right]\{\phi\} \\
\Rightarrow\left[K_{n e t}\right]\{u\} & =\left\{F_{1}\right\}+\left[K_{u \phi}\right]\{\phi\}
\end{aligned}
$$

In the present buckling analysis, von Karman type of nonlinearity is used. Eq. (5) shows the prebuckling equilibrium. In the next step geometric stiffness matrix $\left[K_{G}\right]$ associated with the membrane forces is computed (See Zienkiewicz (1971) for details). The critical buckling load is calculated by solving the linear eigenvalue problem:

$$
\left[K_{n e t}+\lambda K_{G}\right]\{u\}=0
$$

Here $\left[K_{u u}\right],\left[K_{c}\right],\left[K_{G}\right],\left[K_{u \Phi}\right]$ after assembling represents the generalized global stiffness matrices corresponding to mechanical degrees of freedom, additional constraints, instability, and electromechanical coupling. While investigating the bending and buckling behaviour in hygro-thermal environment, the stressstrain relations are modified incorporating the thermal and moisture coefficients (See Sreehari and Maiti, 2015a for details).

\section{Effect of Internal Flaw}

By considering a parameter, the anisotropic damage is parametrically incorporated into the buckling formulation. This parameter is essentially a representation of reduction in effective area and is given by

$$
\Gamma_{i}=\frac{A_{i}-A_{i}^{*}}{A_{i}}
$$

where $A_{i}{ }^{*}$ is the effective area (with unit normal) after damage and $i$ denotes the three orthogonal directions. For a thin plate, only $\Gamma_{1}$ and $\Gamma_{2}$ need to be considered. $\Gamma_{1}$ represents the damage in the direction of the fibre while $\Gamma_{2}$ refers to orthogonal damage (in same plane). The effects of a region of damage are introduced by the use of an idealized model having a reduction in the elastic property in the damage zone. This method which parametrically models damage in any anisotropic material was proposed by Valliappan et al. (1990) and the following relationship between the damaged stress tensor and the undamaged stress tensor was established assuming that the internal forces acting on any damaged section are same as the ones before damage (Valliappan et al., 1990),

$$
\left\{\sigma^{*}\right\}=[\Psi]\{\sigma\}
$$

where $[\Psi]$ is a transformation matrix. This matrix relate a damaged stress-strain matrix with an undamaged one, $\left[H^{*}\right]^{-1}=[\Psi]^{T}[H]^{-1}[\Psi]$. The stress-strain relation can be given as $\left\{\sigma^{*}\right\}=\left[H^{*}\right]\{\varepsilon\}$ for a zone of damage, i.e., it retains its basic form as that of undamaged region and (except incorporation of these parameters) the computations can be proceeded as in the undamaged formulation. This method which parametrically models damage in any anisotropic material was used recently (Sreehari $e t$ al., 2016) for finding the effects of damage in a smart plate.

\section{Optimization Analyses of Damaged Composite Plate}

The maximum thermal buckling load is computed through a Unified Particle Swarm Optimization (UPSO) method. The PSO is a population-based computation method. The concept of bird flocking is used in developing each solution and is referred to as a particle. Mathematically, the positions of $i^{\text {th }}$ particle $\left(x_{i}\right)$ in a swarm of $S$ particles is a $D$-dimensional search space, provides a candidate solution for the problem. The position and velocity of the particles at $t^{\text {th }}$ iteration can be represented by $x_{i}(t)=\left(x_{i 1}, x_{i 2}, x_{i 3}\right.$, $\left.\ldots \ldots \ldots ., x_{i D}\right)$ and $v_{i}(t)=\left(v_{i l}, v_{i 2,}, v_{i 3 \ldots \ldots . . .,} v_{i D}\right)$; where $i \in S$. Motion of each particle to new positions during the search process is based on the previous best position of itself and the best position so far found by any individual of the population. Here the population and its individuals are referred respectively as swarm and particles. The swarm is updated by velocity and position update. Readers may also consult the detailed formulation of PSO given by authors (Sreehari and Maiti, 2015b). Algorithm will lead to a converged solution after several iterations. A flowchart illustrating the PSO algorithm is shown in Fig. 4. In the present study the UPSO, a variant of PSO, is used in the optimization problems.

\section{Results and Discussion}

\section{Introduction}

Presently, an optimization analyses for composite plates using an UPSO algorithm have been done. The governing equations are solved by using finite element 


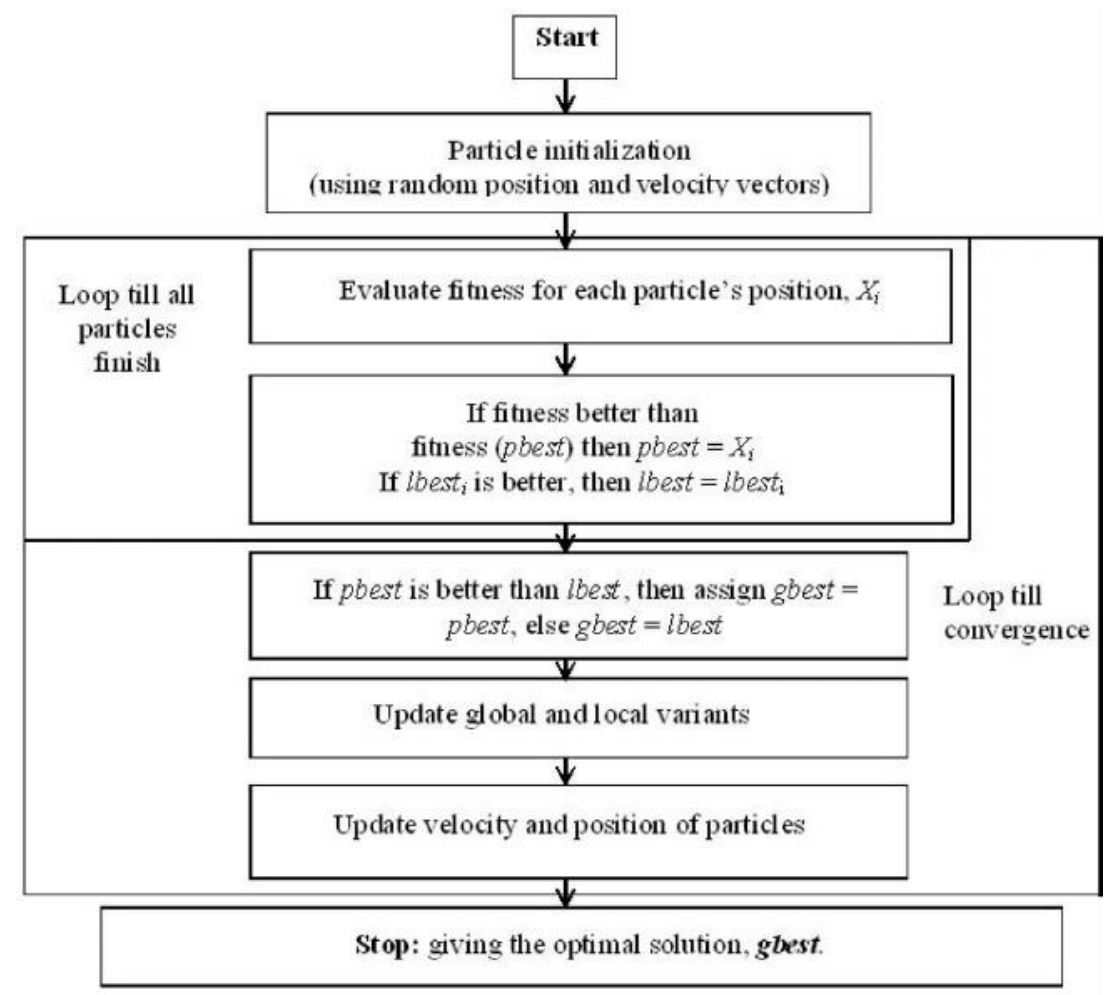

Fig. 4: Schematic diagram illustrating the PSO algorithm

method (FEM). A $\mathrm{C}^{0}$-continuous 8 noded isoparametric element was employed for discretization of the laminated plate. A code is developed for the computer implementation of the finite element formulation in MATLAB environment. Next section presents the comparison studies followed by some important parametric study results.

\section{Validation Studies}

Few validation studies are presented in this section to convey the efficiency of present model. As shown in Fig. 5, analysis is conducted with side to thickness ratio varying from 10 to 100 using FSDT and IHSDT for validation of results. For this study, we have taken a (0/90) s laminated composite plate with simply supported at all ends subjected to uniaxial inplane loads. Similar comparison analysis with biaxial inplane loads is done and results are shown in Fig. 6. It is noticed that nondimensional critical loads are larger for uniaxial loading. And as thickness decreases, the nondimensional buckling load increases. Numerical results for a smart plate are validated and shown in Table 1. The non-dimensionalised critical buckling load is presented when piezolayers are attached to top and

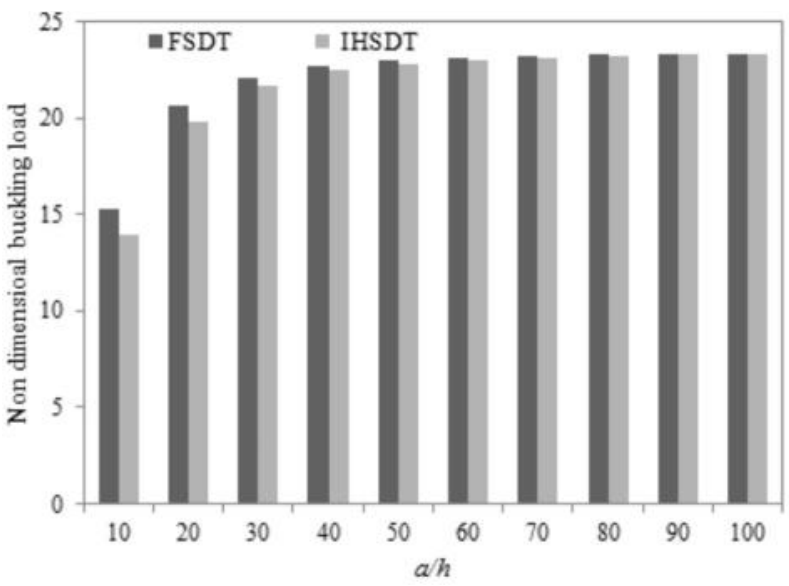

Fig. 5: Buckling load using FSDT and IHSDT (for uniaxial loading)

bottom of the composite plate with a net $a / h$ ratio 10 . The detailed validation studies are presented in Sreehari et al. (2016). Table 2 presents a comparison study after including the effects of damage for a simple plate. The present results are compared with the results obtained by Prabhakara and Datta (1993). The buckling coefficients for a plate with 3 different aspect ratios are calculated and matched with those of reference as shown in Table 2, and validates the finite 


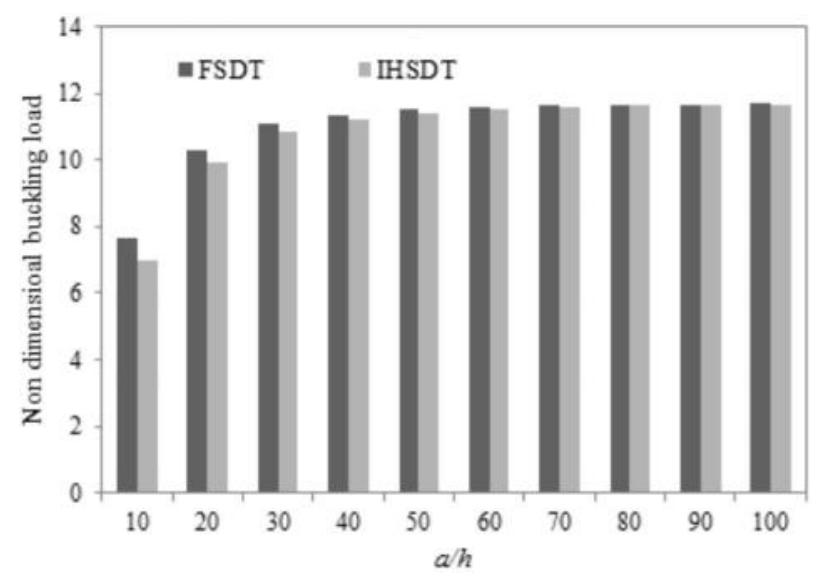

Fig. 6: Buckling load using FSDT and IHSDT (for biaxial loading)

element formulation incorporating damage and the methodology. So, it is observed from these comparison results that present finite element model predicts the bending and buckling behavior quite accurately.

\section{Parametric Studies}

Analysis is done for symmetric (0/90/90/0) laminates under uniaxial compression for undamaged and damaged cases. Here we have used a 10x10 meshing to discretize the whole plate and the center 4 elements are considered to have a mild damage. The capability of piezopatches in controlling the deflection of the plate was analyzed and found as in the Fig. 7. In this validation study, the piezoelectric layers are attached firmly to top and bottom surfaces of the laminated composite plate. The results were found for different voltages $(0 \mathrm{~V}, 100 \mathrm{~V}$ and $150 \mathrm{~V})$ in piezo-layer. It is found that the piezolayers under the application of

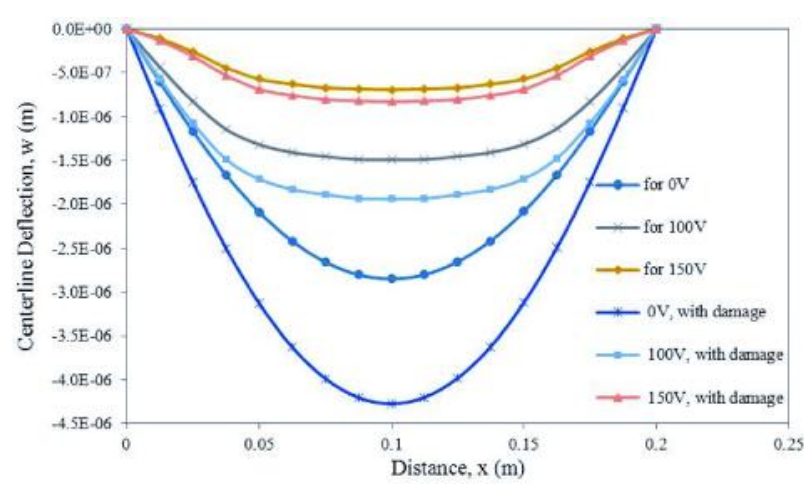

Fig. 7: Deflection control under voltages of SS plate for UDL of $20000 \mathrm{Nm}^{-2}$
Table 1: Nondimensional critical buckling load validation

\begin{tabular}{lcc}
\hline Source & $\begin{array}{c}\text { Nondimensional critical } \\
\text { buckling load of } \\
\text { smart plate }\end{array}$ & $\begin{array}{c}\text { Error } \\
\text { percentage }\end{array}$ \\
\hline FSDT & 14.87 & -4.2 \\
Present (IHSDT) & 15.02 & -3.2 \\
Wankhade and Bajoria (2012) & 15.51 & - \\
\hline
\end{tabular}

Table 2: Buckling coefficients of a damaged composite plate for 3 different aspect ratios

\begin{tabular}{lccc}
\hline AR & Present & $\begin{array}{c}\text { Prabhakara and } \\
\text { Datta (1993) }\end{array}$ & Variation \\
\hline 0.8 & 3.48 & 3.59 & $3.06 \%$ \\
1 & 4.32 & 4.45 & $2.92 \%$ \\
1.6 & 1.71 & 1.77 & $3.38 \%$ \\
\hline
\end{tabular}

voltages were capable of controlling the center point deflection. Central deflection increases in plates having an internal flaw due to its decreased stiffness. It is observed that to reduce the effects of flaw, a smart plate can be used instead of a simple plate.

Capability of centrally located PFCPs in controlling the deflection is studied in the next analysis. PFCPs attached firmly to the top surface (in an area of the centre 4 elements for a plate with $10 \times 10$ mesh size) of the laminated composite plateis considered. Material properties used for PFCs were $E=63 \mathrm{GPa}$, $\nu=0.31, \rho=7600 \mathrm{kgm}^{-3} \quad K_{11}=K_{22}=15.3 \times 10^{-9}$ $\mathrm{Fm}^{-1}, \quad K_{33}=15 \times 10^{-9} \mathrm{Fm}^{-1}, e_{11}=14.14 \mathrm{C} / \mathrm{m}^{2}, e_{21}=$ $-3.34 \mathrm{C} / \mathrm{m}^{2}, e_{24}=10.79 \mathrm{C} / \mathrm{m}^{2}$. The thermal expansion coefficients used were $\alpha_{1}=2 \times 10^{-60} \mathrm{C}^{-1}, \alpha_{2} / \alpha_{1}=10$. Here also we have used a mesh size of $10^{*} 10$ to discretize the whole plate and the center 4 elements are considered to have a mild damage. The capability of centrally located PFCPs in controlling the deflection of plate (PFCP/0/90/0/90/90/0/90/0) was analysed in Fig. 8. Firstly, we examined the effect of temperature increment $(\Delta \mathrm{T}=5)$ and then we considered both the effect of temperature increment and the effect of internal flaw on deflection. It is observed that the central deflection increases in plates having an internal flaw due to its decreased stiffness and due to thermal effects. Then we considered the application of $5 \mathrm{~V}$ on the PFC patches. It is observed that by applying voltage on PFC patches, deformations of the plate with damage got counteracted to large extent and 


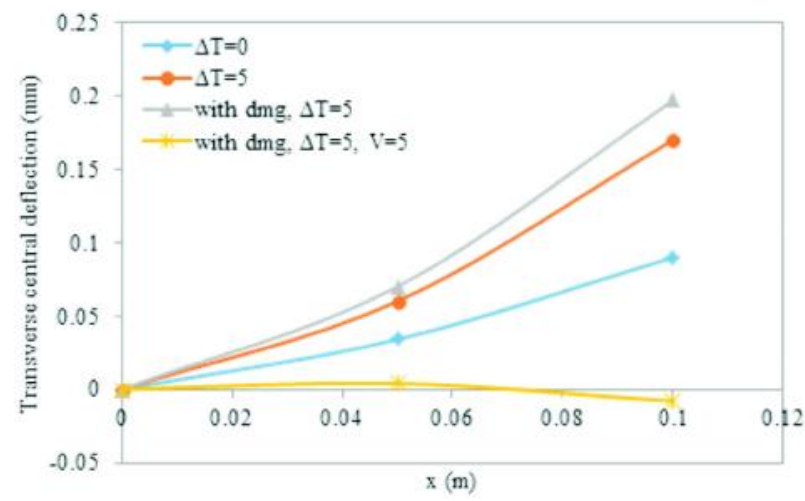

Fig. 8: Deflection control under voltages of a cantilever plate (PFCP/0/90/0/90/90/0/90/0) with PFCP

failure due to thermal environment is also prevented. Further, results from similar analysis for various laminates like (PFCP/0/0/0/0/90/90/90/90), (PFCP/45/ 45/-45/-45/-45/-45/45/45), and (PFCP/45/45/-45/-45/ 45/45/-45/-45) are shown respectively in Figs. 9, 10 and 11 . Thus the ability and efficiency of PFCPs to overcome the degradation in strength of composites is proved.

A composite plate with properties as in above example is considered now for finding the best location for the piezopatches. A $(0 / 90 / 90 / 0)$ plate with segmented piezopatches (PFCPs) on the top is considered. The UPSO algorithm related parameters used here are: Cognitive parameter, $C_{1}=2.05$, Social parameter, $C_{2}=2.05$ and Constriction factor, $R=$ 0.7298. Clerc (2002) had explained in detail the constriction factor $R$, that increases PSO's ability to constrain and control velocities and explained its calculation. Literatures (Premalatha and Natarajan,

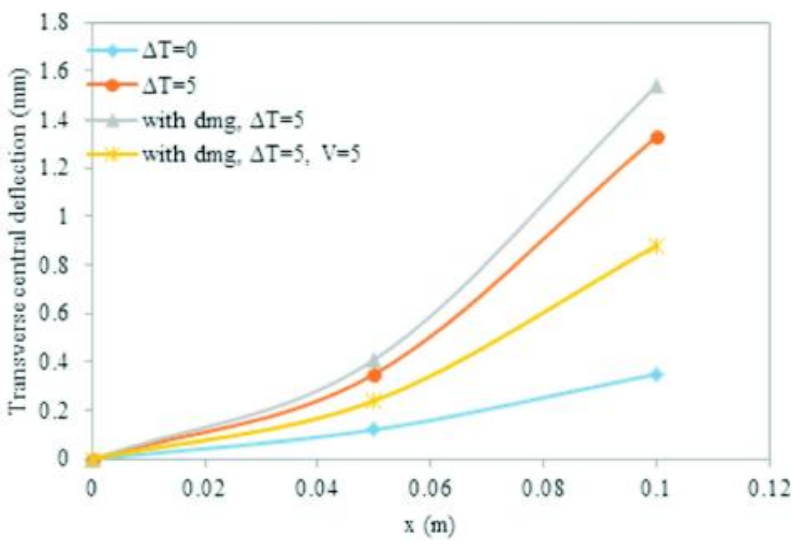

Fig. 9: Deflection control under voltages of a cantilever plate (PFCP/0/0/0/0/90/90/90/90) with PFCP

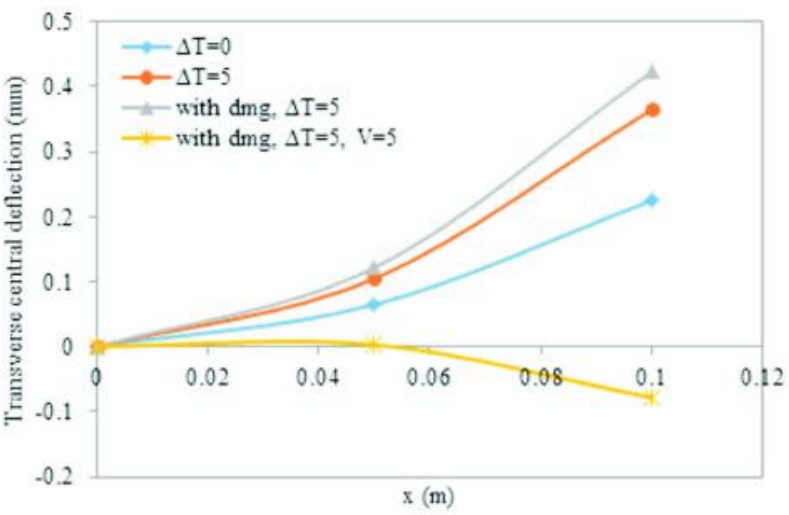

Fig. 10: Deflection control under voltages of a cantilever plate (PFCP/45/45/-45/-45/-45/-45/45/45) with PFCP

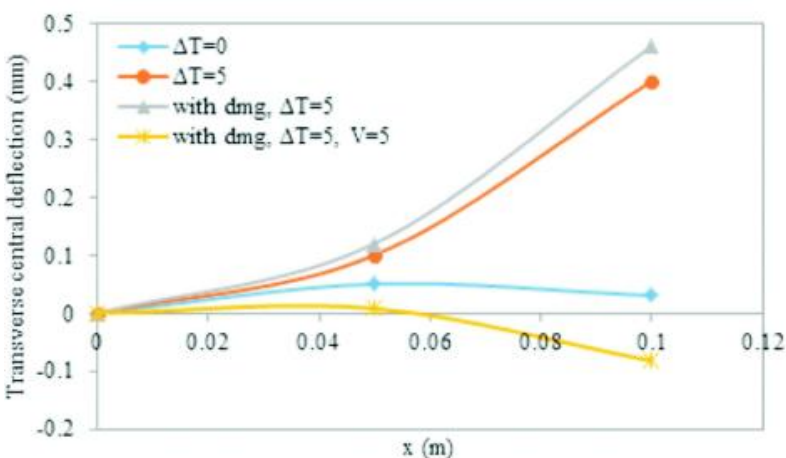

Fig. 11: Deflection control under voltages of a cantilever plate (PFCP/45/45/-45/-45/45/45/-45/-45) with PFCP

2009; Sumathi and Surekha, 2010) suggested that it would be better to choose $C_{1}=C_{2}=2.05$ which shown an overall better performance of PSO. Even though there are a lot of locations possible for these PFCPs over the substrate, the locations are restricted for maximizing the buckling load. The size of PFCP is equivalent to size of a single element in the finite element mesh. To find out the best positions of piezopathces UPSO algorithm is employed. The number of piezopatches to be employed for forming optimized pattern is given in the code during the initial steps. Then the optimization algorithm searches and finds the best locations for the patches. The best location corresponds to the location having maximum value of fitness function. The fitness function here is the nondimensional critical buckling temperature. The fitness function of the UPSO algorithm is found each time by doing the finite element analysis for each particle. The value of nondimensional buckling load from finite element analysis loop is returned to UPSO algorithm each time. Figs. 12 and 13 show the optimal 
locations of 4 PFCPs for simply supported and cantilever boundary conditions respectively. The optimized patch locations are near the central damage in a simply supported plate while they are near the fixed end in a cantilever plate. Similarly, Figs. 14 and 15 show the optimal locations of 8 PFCPs for simply supported and cantilever boundary conditions respectively. The critical buckling temperatures obtained for the cases presented in Figs. 12 and 14 (cases with simply supported boundary condition) are shown in Table 3. It is observed from Table 3 that the critical buckling temperature for a composite plate with damage is lesser than that of undamaged plate. Then as the PFCPs are placed in their optimized locations, an increment in critical temperature is

\begin{tabular}{|c|c|c|c|c|c|c|c|c|c|}
\hline 91 & 92 & 93 & 94 & 95 & 96 & 97 & 98 & 99 & 100 \\
\hline 81 & 82 & 83 & 84 & 85 & 86 & 87 & 88 & 89 & 90 \\
\hline 71 & 72 & 73 & 74 & 75 & 76 & 77 & 78 & 79 & 80 \\
\hline 61 & 62 & 63 & 64 & 65 & 66 & 67 & 68 & 69 & 70 \\
\hline 51 & 52 & 53 & 54 & 55 & 56 & 57 & 58 & 59 & 60 \\
\hline 41 & 42 & 43 & 44 & 45 & 46 & 47 & 48 & 49 & 50 \\
\hline 31 & 32 & 33 & 34 & 35 & 36 & 37 & 38 & 39 & 40 \\
\hline 21 & 22 & 23 & 24 & 25 & 26 & 27 & 28 & 29 & 30 \\
\hline 11 & 12 & 13 & 14 & 15 & 16 & 17 & 18 & 19 & 20 \\
\hline 1 & 2 & 3 & 4 & 5 & 6 & 7 & 8 & 9 & 10 \\
\hline
\end{tabular}

Fig. 12: Optimal positioning of 4 piezo patches in a simply supported plate

\begin{tabular}{|c|c|c|c|c|c|c|c|c|c|}
\hline 91 & 92 & 93 & 94 & 95 & 96 & 97 & 98 & 99 & 100 \\
\hline 81 & 82 & 83 & 84 & 85 & 86 & 87 & 88 & 89 & 90 \\
\hline 71 & 72 & 73 & 74 & 75 & 76 & 77 & 78 & 79 & 80 \\
\hline 61 & 62 & 63 & 64 & 65 & 66 & 67 & 68 & 69 & 70 \\
\hline 51 & 52 & 53 & 54 & 55 & 56 & 57 & 58 & 59 & 60 \\
\hline 41 & 42 & 43 & 44 & 45 & 46 & 47 & 48 & 49 & 50 \\
\hline 31 & 32 & 33 & 34 & 35 & 36 & 37 & 38 & 39 & 40 \\
\hline 21 & 22 & 23 & 24 & 25 & 26 & 27 & 28 & 29 & 30 \\
\hline 11 & 12 & 13 & 14 & 15 & 16 & 17 & 18 & 19 & 20 \\
\hline 1 & 2 & 3 & 4 & 5 & 6 & 7 & 8 & 9 & 10 \\
\hline
\end{tabular}

Fig. 13: Optimal positioning of 4 piezo patches in a cantilever plate

\begin{tabular}{|c|c|c|c|c|c|c|c|c|c|}
\hline 91 & 92 & 93 & 94 & 95 & 96 & 97 & 98 & 99 & 100 \\
\hline 81 & 82 & 83 & 84 & 85 & 86 & 87 & 88 & 89 & 90 \\
\hline 71 & 72 & 73 & 74 & 75 & 76 & 77 & 78 & 79 & 80 \\
\hline 61 & 62 & 63 & 64 & 65 & 66 & 67 & 68 & 69 & 70 \\
\hline 51 & 52 & 53 & 54 & 55 & 56 & 57 & 58 & 59 & 60 \\
\hline 41 & 42 & 43 & 44 & 45 & 46 & 47 & 48 & 49 & 50 \\
\hline 31 & 32 & 33 & 34 & 35 & 36 & 37 & 38 & 39 & 40 \\
\hline 21 & 22 & 23 & 24 & 25 & 26 & 27 & 28 & 29 & 30 \\
\hline 11 & 12 & 13 & 14 & 15 & 16 & 17 & 18 & 19 & 20 \\
\hline 1 & 2 & 3 & 4 & 5 & 6 & 7 & 8 & 9 & 10 \\
\hline
\end{tabular}

Fig. 14: Optimal positioning of 8 piezopatches in a simply supported plate

\begin{tabular}{|c|c|c|c|c|c|c|c|c|c|}
\hline 91 & 92 & 93 & 94 & 95 & 96 & 97 & 98 & 99 & 100 \\
\hline 81 & 82 & 83 & 84 & 85 & 86 & 87 & 88 & 89 & 90 \\
\hline 71 & 72 & 73 & 74 & 75 & 76 & 77 & 78 & 79 & 80 \\
\hline 61 & 62 & 63 & 64 & 65 & 66 & 67 & 68 & 69 & 70 \\
\hline 51 & 52 & 53 & 54 & 55 & 56 & 57 & 58 & 59 & 60 \\
\hline 41 & 42 & 43 & 44 & 45 & 46 & 47 & 48 & 49 & 50 \\
\hline 31 & 32 & 33 & 34 & 35 & 36 & 37 & 38 & 39 & 40 \\
\hline 21 & 22 & 23 & 24 & 25 & 26 & 27 & 28 & 29 & 30 \\
11 & 12 & 13 & 14 & 15 & 16 & 17 & 18 & 19 & 20 \\
1 & 2 & 3 & 4 & 5 & 6 & 7 & 8 & 9 & 10 \\
\hline
\end{tabular}

Fig. 15: Optimal positioning of 8 piezopatches in a cantilever plate

Table 3. Enhanced critical buckling temperatures $\left({ }^{0} \mathrm{C}\right)$ by using PFCPs at optimized locations for a composite plate with damage

\begin{tabular}{lccc}
\hline $\begin{array}{l}\text { Undamaged } \\
\text { case }\end{array}$ & $\begin{array}{c}\text { Damaged } \\
\text { case }\end{array}$ & $\begin{array}{c}\text { Damaged } \\
\text { case } \\
\text { (with 4 PFCPs) }\end{array}$ & $\begin{array}{c}\text { Damaged } \\
\text { case } \\
\text { (with 8 PFCPs) }\end{array}$ \\
\hline 72 & 67.3 & $68.6($ at $0 \mathrm{~V})$ & $69.9($ at $0 \mathrm{~V})$ \\
- & - & $70.8($ at $50 \mathrm{~V})$ & 72.3 (at 50 V) \\
\hline
\end{tabular}

obtained. And the increment is very large when a 50 $\mathrm{V}$ was applied on PFCPs. Thus, once the actuator patches are optimally placed, the effects due to damage are suppressed and the bending and buckling capacity of composite laminates are enhanced. In addition to the best possible performance, huge 
reduction in weight of smart material happened due to the placement of segmented PFCPs (instead of piezo layer) to overcome the effects of damage and hygrothermal environment in the bending and buckling analysis for a composite plate. It seems to have major practical significance and these applications prove the contribution of present investigation to be of realistic nature.

\section{Conclusion}

This work has been done for bending and buckling analyses of laminated composite plate equipped with PFCPs. The governing equations are solved by using finite element method considering an eight noded isoparametric element and using inverse hyperbolic shear deformation theory. The present theory helps to analyze complex problems under less computational complexity. The results got are quite accurate and show excellent performance of the present formulation. Validation of the current analysis showed desired outputs. The subsequent important conclusions are noted from the present investigation,

- The capability of centrally located PFCPs in controlling the deflection of plate was analysed for symmetric and anti-symmetric composite plates.

- It is observed that employing a centrally located PFC patch above top surface of composite plate reduces the hygrothermal effects and effects of internal flaw.

\section{References}

Aydogdu M (2009) A new shear deformation theory for laminated composite plates Compos Struct 89 94-101

Banks A, Vincent J and Anyakoha C (2007) A review of particle swarm optimization Part I: background and development Natural Computing 6 467-484

Banks A, Vincent J and Anyakoha C (2008) A review of particle swarm optimization. Part II: hybridisation, combinatorial, multicriteria and constrained optimization and indicative applications Natural Computing 7 109-124

Bent AA (1997) Active Fiber Composites for Structural Actuation Ph. D. Thesis, Massachusetts Institute of Technology, Cambridge

Chandrashekhara K and Bhatia K (1993) Active buckling control
- It is also noticed that by applying voltage on PFC patches, deformations of the plate with damage got counteracted to large extent and failure due to hygrothermal environment is prevented.

- For optimum designs, the structures should be capable of withstanding maximum possible forces acting on them. Also the structures should be able to overcome the effects of small damages occurring in them. To enhance this capability we can use smart materials along with structural components in order to make them withstand more forces than what they are expected to.

- In addition to other reasons, if we use segmented PFCPs over a composite substrate, considerable weight reduction is obtained.

- The present work provides the optimal placement of PFCP actuators. Investigations are carried out on application of optimized piezo locations in strengthening structures, thereby controlling the deformations (due to external forces or caused as an effect of a flaw present in the system) and increasing the critical buckling temperatures.

- It is observed from this work that UPSO is a very promising optimization technique and can be successfully applied to find the maximized buckling temperatures of smart structures.

of smart composite plates-finite-element analysis Smart Mater Struct 2 31-39

Clerc M and Kennedy J (2002) The particle swarm-explosion, stability, and convergence in a multidimensional complex space IEEE Trans Evol Comput 6 58-73

Crawley and Luis (1987) Use of piezoelectric actuators as elements of intelligent structures AIAA J 25 1373-1385

Franco Correia V M, Mota Soares C M and Mota Soares C A (2003) Buckling optimization of composite laminated adaptive structures J Compos Struct 62 315-321

Frecker M I (2003) Recent advances in optimization of smart structures and actuators J Intell Matr Syst Struct 14 207216

Grover N, Singh B N and Maiti D K (2013) New nonpolynomial 
shear-deformation theories for structural behavior of laminated-composite and sandwich plates AIAA J 51 18611871

Hwang W S and Park H C (1993) Finite element modeling of piezoelectric sensors and actuators AIAA J 31 930-937

Jeng-Shian Chang and Yuh-Pao Huang (1991) Nonlinear analysis of composite antisymmetric angle-ply under uniform temperature field J Comput Struct 40 857-869

Karama M, Afaq K S and Mistou S (2009) A new theory for laminated composite plates Proc IMechE 223 Part L: J Mater: Des Appl

Kennedy J and Eberhart R (1995) Particle swarm optimization In: Proc. IEEE Int Conf on Neural Networks (Perth) IEEE Service Center 4, Piscataway, NJ, 1942-1948

Maiti D K and Sinha P K (2011) Analysis of smart laminated composites employing piezo embedded super element Procedia Eng 14 3268-3276

Mantari J L, Oktem A S and Soares C G (2012a) A new trigonometric shear deformation theory for isotropic, laminated composite and sandwich plates Int J Solids Struct 49 43-53

Mantari J L, Oktem A S and Soares C G (2012b) A new higher order shear deformation theory for sandwich and composite laminated plates Compos B 43 1489-1499

Meiche N E, Tounsi A, Zlane N, Mechab I and Bedia E A A (2011) A new hyperbolic shear deformation theory for buckling and vibration of functionally graded sandwich plate Int J Mech Sci 53 237-247

Meyers C A and Hyer M W (1991) Thermal buckling and postbuckling of symmetrically laminated composite plates $J$ Therm Stresses 14 519-540

Mohan S C, Amit Yadav, Maiti D K and Damodar Maity (2014) A comparative study on crack identification of structures from the changes in natural frequencies using GA and PSO Eng Comput 31 1514-1531

Noor A K, James H Starnes Jr and Jeanne M Peters (1993) Thermo-mechanical buckling and postbuckling of multilayered composite panels J Compos Struct 23 233-251

Parsopoulos K E and Vrahatis M N (2002) Recent approaches to global optimization problems through Particle Swarm Optimization Nat Computing 1 235-306

Pidaparti R M V (1997) Free vibration and flutter of damaged composite panels Compos Struct 38 477-481

Prabhakara D L and Datta P K (1993) Vibration and static stability characteristics of rectangular plates with a localized flaw Comput Struct 49 825-836

Pratihar D K (2008) Soft computing Narosa publishing house Pvt
Ltd, NewDelhi

Premalatha K and Natarajan A M (2009) Hybrid PSO and GA for global maximization Int J Open Problems Comput Math 2 597-608

Rahul R and Datta P K (2013) Static and dynamic characteristics of thin plate like beam with internal flaw subjected to inplane harmonic load Int J Aeronautical and Space Sci 14 19-29

Rao S S (2009) Engineering Optimization: Theory and Practice 4th ed John Wiley \& Sons, Inc

Ray M C, Bhattacharyya R and Samanta B (1994) Static analysis of an intelligent structure by finite element method Comput Struct 52 617-631

Reddy J N (1999) On laminated composite plates with integrated sensors and actuators Eng Struct 21 568-593

Samanta B, Ray M C and Bhattacharyya R (1996) Finite element model for active control of intelligent structures AIAA J 34 1885-1893

Singha M K, Ramchandra L S and Bandyopadhyay J N (2001) Thermal postbuckling analysis of laminated composite plate J Compos Struct $\mathbf{5 4}$ 453-458

Sohn H, Farrar R C, Hemez M F, Shunk D D, Stinemates W S, Nadler R B and Czamecki J J (2004) A review of structural health monitoring literature: 1996-2001, Los Alamos National Laboratory Report No. LA-13976-MS Los Alamos, New Maxico

Sreehari V M and Maiti D K (2015a) Buckling and post buckling analysis of laminated composite plates in hygrothermal environment using an Inverse hyperbolic shear deformation theory J Compos Struct 129 250-255

Sreehari V M and Maiti D K (2015b) Optimization of damaged composite plates under buckling and post buckling condition in hygrothermal environment employing an inverse hyperbolic shear deformation theory In: 56th AIAA/ ASME/ASCE/AHS/ASC Struct, Struct Dyn and Matr Conf, AIAA Sci Tech Forum, Florida. doi: 10.2514/6.2015-144

Sreehari V M, Linju Joseph George and Maiti D K (2016) Bending and buckling analysis of smart composite plates with and without internal flaw using an inverse hyperbolic shear deformation theory J Compos Struct 138 64-74

Sumathi S and Surekha P (2010) Computational intelligence paradigms- Theory and applications using MATLAB. CRC Press

Tauchert T R (1987) Thermal buckling of thick antisymmetric angle-ply laminates $J$ Therm Stresses 10 113-124

Tauchert T R and Huang N N (1987) Thermal buckling of symmetric angle-ply laminated plates In: 4th Int Conf on 
Compos Struct Paisley, UK, 1424-1435

Thangaratnam K R, Palaninathan R and Ramachandran (1989)

Thermal buckling of composite laminated plates J Comput Struct 32 1117-1124

Valliappan S, Murti V and Zhang Wohua (1990) Finite element analysis of anisotropic damage mechanics problems Eng Fract Mech 35 1061-1071

Wankhade R L and Bajoria K M (2012) Stability of simply supported smart piezolaminated composite plate using finite element method In: Proc of the Int Confon Advances in Aeronautical and Mech Eng ISBN:978-981-07-2683-6

Zhang W, Yunmin C and Yi J (2001) Effects of symmetrisation of net-stress tensor in anisotropic damage models Int J Fract $109345-363$

Zhou Y L (2001) Modeling of piezoelectric composite laminates using a third-order plate theory In: Int Offshore and Polar Eng Conf 4 131-139

Zienkiewicz O C (1971) The Finite Element Method, 3rd ed. Tata McGraw-Hill publishing. 